\title{
A representation basis for the quantum integrable spin chain associated with the $\mathrm{su}(3)$ algebra
}

\author{
Kun Hao, ${ }^{a}$ Junpeng Cao, ${ }^{b, c}$ Guang-Liang Li, ${ }^{d}$ Wen-Li Yang, ${ }^{a, e, 1}$ Kangjie Shi ${ }^{a}$ \\ and Yupeng Wang ${ }^{b, c, 1}$ \\ ${ }^{a}$ Institute of Modern Physics, Northwest University, \\ Xian 710069, China \\ ${ }^{b}$ Beijing National Laboratory for Condensed Matter Physics, \\ Institute of Physics, Chinese Academy of Sciences, \\ Beijing 100190, China \\ ${ }^{c}$ Collaborative Innovation Center of Quantum Matter, \\ Beijing, China \\ ${ }^{d}$ Department of Applied Physics, Xian Jiaotong University, \\ Xian 710049, China \\ ${ }^{e}$ Beijing Center for Mathematics and Information Interdisciplinary Sciences, \\ Beijing 100048, China \\ E-mail: haoke72@163.com, junpengcao@iphy.ac.cn, \\ leegl@mail.xjtu.edu.cn, wlyang@nwu.edu.cn, kjshi@nwu.edu.cn, \\ yupeng@iphy.ac.cn
}

ABSTRACT: An orthogonal basis of the Hilbert space for the quantum spin chain associated with the $\mathrm{su}(3)$ algebra is introduced. Such kind of basis could be treated as a nested generalization of separation of variables ( $\mathrm{SoV}$ ) basis for high-rank quantum integrable models. It is found that all the monodromy-matrix elements acting on a basis vector take simple forms. With the help of the basis, we construct eigenstates of the su(3) inhomogeneous spin torus (the trigonometric $\mathrm{su}(3)$ spin chain with antiperiodic boundary condition) from its spectrum obtained via the off-diagonal Bethe Ansatz (ODBA). Based on small sites (i.e. $N=2$ ) check, it is conjectured that the homogeneous limit of the eigenstates exists, which gives rise to the corresponding eigenstates of the homogenous model.

Keywords: Bethe Ansatz, Lattice Integrable Models

ArXiv EPrint: 1601.04771

\footnotetext{
${ }^{1}$ Corresponding author.
} 


\section{Contents}

1 Introduction 1

$2 \operatorname{su}(n)$ spin torus and its spectrum 2

3 Nested SoV basis $\quad 6$

4 Eigenstates of the transfer matrix $\quad 10$

5 Conclusions 12

$\begin{array}{ll}\text { A Exchange relations } & 13\end{array}$

$\mathrm{B} \operatorname{su}(n)$ case $\quad 14$

$\begin{array}{ll}\text { C Proof of the operator decomposition } & 14\end{array}$

$\begin{array}{ll}\text { D Proof of (4.7) } & 15\end{array}$

\section{Introduction}

Quantum integrable system has played an important role in understanding the physical contents of the planar $\mathcal{N}=4$ super-symmetric Yang-Mills (SYM) theory and the planar AdS/CFT [1, 2] (see also references therein). Moreover, it has also provided valuable insight into important universality class in condensed matter physics [3] and cold atom systems [4]. In the past several decades, the integrable quantum spin chains with U(1)symmetry (with periodic boundary or with diagonal open boundaries [5]) and with some constrained open boundaries [6-21] have been extensively studied by various Bethe ansatz methods for a finite lattice and by the vertex operator method [22] in an infinite or a half-infinite lattice [23-28].

Very recently, an important progress has been achieved in solving the eigenvalue problem of integrable models without U(1)-symmetry [29] (i.e., the off-diagonal Bethe Ansatz (ODBA), for comprehensive introduction we refer the reader to [30]). Several long-standing models [29, 31-36] have since been solved. It should be noted that besides ODBA [37, 38] some other methods such as the q-Onsager algebra method [39-42], the separation of variables (SoV) method [43-49] and the modified algebraic Bethe ansatz method [50-53] were also used to obtain the eigenstates of the XXZ spin chains with generic boundary conditions. Remarkably, ODBA allows us to obtain eigenvalues of the U(1)-broken models associated with higher-rank algebras such as the $\operatorname{su}(n)$ spin chain with generic integrable boundary 
fields [35], the Izergin-Korepin model ${ }^{1}$ with generic boundary conditions [36], the Hubbard model [34] and the supersymmetric $t-J$ model [54] with unparallel boundary fields, and the open chain related to AdS/CFT [55]. However, the corresponding eigenstates for these models are still missing.

According to Liouville's theorem, a key feature of integrable models is that their variables are completely separable. This concept was generalized to quantum integrable models by Sklyanin [56-58] and provided a promising approach to construct eigenstates of quantum integrable models without U(1)-symmetry. Nevertheless, Sklyanin's SoV procedure has only succeeded for some rank-one quantum integrable models and a proper SoV scheme for the high-rank quantum integrable models is still absent. The main task of the present paper is to propose a nested $\mathrm{SoV}$ basis for the $\mathrm{su}(n)$ spin chain model. As an example of application, we construct exact eigenstates of the $\mathrm{su}(3)$ spin torus (i.e., the trigonometric $\mathrm{su}(3)$ spin chain with anti-periodic boundary condition), an archetype high-rank quantum integrable model without highest weight reference state, based on its spectrum recently obtained in [59] via ODBA.

The paper is organized as follows. Section 2 serves as an introduction to our notations for the inhomogeneous $\mathrm{su}(n)$ spin torus and its spectrum. In section 3, we introduce a nested SoV basis of the Hilbert space of the su(3) spin chain. It is found that the actions of the monodromy matrix elements on a basis vector have no compensating exchange terms on the level of the local operators (i.e., polarization free) and therefore become drastically simple. In section 4 , with the help of the basis, as an example, we construct eigenstates of the transfer matrix for the su(3) spin torus from its spectrum obtained via ODBA [59]. Concluding remarks are given in section 5. Some detailed technical proofs are given in appendices A-D.

\section{$2 \quad \mathrm{su}(n)$ spin torus and its spectrum}

Let $\mathbf{V}$ denote an $n$-dimensional linear space with an orthonormal basis $\{|i\rangle \mid i=1, \cdots, n\}$. We introduce the Hamiltonian $H$ as follows:

$$
H=\sum_{j=1}^{N} h_{j, j+1},
$$

where $N$ is the number of sites and $h_{j, j+1}$ is the local Hamiltonian given by

$$
h_{j, j+1}=\left.\frac{\partial}{\partial u}\left\{P_{j, j+1} R_{j, j+1}(u)\right\}\right|_{u=0} .
$$

Here $P_{j, j+1}$ is the permutation operator on the tensor space and the $R$-matrix $R(u) \in$ $\operatorname{End}(\mathbf{V} \otimes \mathbf{V})$ is the trigonometric $R$-matrix associated with the quantum group [60]

\footnotetext{
${ }^{1}$ It is a model beyond A type.
} 
$U_{q}(\widehat{\operatorname{su}(n)})$, which was first proposed by Perk and Shultz [61] and further studied in $[62-66]^{2}$

$$
\begin{aligned}
R(u)= & \sinh (u+\eta) \sum_{k=1}^{n} E^{k, k} \otimes E^{k, k}+\sinh u \sum_{k \neq l}^{n} E^{k, k} \otimes E^{l, l} \\
& +\sinh \eta\left(\sum_{k<l}^{n} e^{\frac{n-2(l-k)}{n} u}+\sum_{k>l}^{n} e^{-\frac{n-2(k-l)}{n} u}\right) E^{k, l} \otimes E^{l, k},
\end{aligned}
$$

where the $n^{2}$ fundamental matrices $\left\{E^{k, l} \mid k, l=1, \cdots, n\right\}$ are all $n \times n$ matrices with matrix entries $\left(E^{k, l}\right)_{\beta}^{\alpha}=\delta_{\alpha}^{k} \delta_{\beta}^{l}$ and $\eta$ is the crossing parameter. The $R$-matrix satisfies the quantum Yang-Baxter equation (QYBE)

$$
R_{12}\left(u_{1}-u_{2}\right) R_{13}\left(u_{1}-u_{3}\right) R_{23}\left(u_{2}-u_{3}\right)=R_{23}\left(u_{2}-u_{3}\right) R_{13}\left(u_{1}-u_{3}\right) R_{12}\left(u_{1}-u_{2}\right),
$$

and possesses the properties:

Initial condition:

$$
R_{12}(0)=\sinh \eta P_{1,2},
$$

$$
\text { Unitarity: }
$$

$$
R_{12}(u) R_{21}(-u)=\rho_{1}(u) \times \mathrm{id},
$$

$$
\rho_{1}(u)=-\sinh (u+\eta) \sinh (u-\eta),
$$

Crossing-unitarity: $\quad R_{12}^{t_{1}}(u) R_{21}^{t_{1}}(-u-n \eta)=\rho_{2}(u) \times \mathrm{id}, \quad \rho_{2}(u)=-\sinh u \sinh (u+n \eta)$,

Fusion conditions:

$$
R_{12}(-\eta)=-2 \sinh \eta P_{1,2}^{(-)} \text {. }
$$

Here $R_{21}(u)=P_{1,2} R_{12}(u) P_{1,2} ; P_{1,2}^{(-)}$is the q-deformed anti-symmetric project operator [59] in the tensor product space $\mathbf{V} \otimes \mathbf{V}$; and $t_{i}$ denotes the transposition in the $i$-th space. Here and below we adopt the standard notation: for any matrix $A \in \operatorname{End}(\mathbf{V}), A_{j}$ is an embedding operator in the tensor space $\mathbf{V} \otimes \mathbf{V} \otimes \cdots$, which acts as $A$ on the $j$-th space and as an identity on the other factor spaces; $R_{i j}(u)$ is an embedding operator of $R$-matrix in the tensor space, which acts as an identity on the factor spaces except for the $i$-th and $j$-th ones. For the $\mathrm{su}(3)$ case the $R$-matrix reads

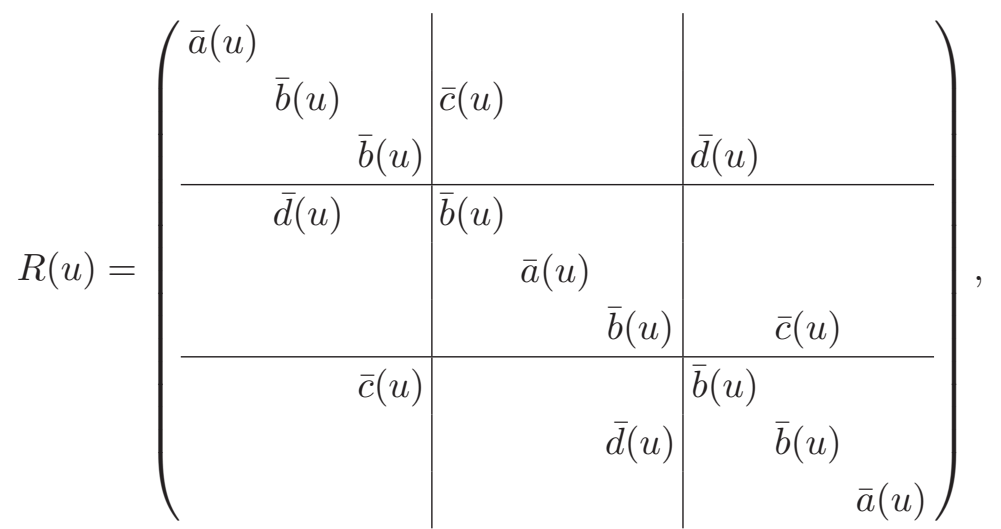

where the matrix elements are

$$
\begin{array}{llrl}
\bar{a}(u) & =\sinh (u+\eta), & \bar{b}(u) & =\sinh u, \\
\bar{c}(u) & =e^{\frac{u}{3}} \sinh \eta, & \bar{d}(u) & =e^{-\frac{u}{3}} \sinh \eta .
\end{array}
$$

\footnotetext{
${ }^{2}$ The $R$-matrix given by (2.3) corresponds to the so-called principal gradation, which is related to the $R$-matrix in homogeneous gradation by some gauge transformation [67].
} 
Let us introduce the $n \times n$ twist matrix $g$

$$
g=\left(\begin{array}{ccc} 
& & \\
1 & & \\
& \ddots & \\
& & 1
\end{array}\right), \quad \text { and } g^{n}=1
$$

For the $\mathrm{su}(3)$ case, it reads

$$
g=\left(\begin{array}{lll}
0 & 0 & 1 \\
1 & 0 & 0 \\
0 & 1 & 0
\end{array}\right), \quad \text { and } g^{3}=1 .
$$

It is found that the $R$-matrix (2.3) is invariant with $g$,

$$
g_{0} g_{0^{\prime}} R_{00^{\prime}}(u) g_{0}^{-1} g_{0^{\prime}}^{-1}=R_{00^{\prime}}(u) .
$$

This property enables us to construct the integrable $\mathrm{su}(n)$ spin torus model [59].

Similar to the $\mathrm{su}(2)$ spin torus (or the XXZ spin chain with anti-periodic boundary condition) [68], the $\operatorname{su}(n)$ spin torus is described by the Hamiltonian $H$ given by (2.1) with anti-periodic boundary conditions

$$
E_{N+1}^{k, l}=g_{1} E_{1}^{k, l} g_{1}^{-1}, \quad k, l=1, \cdots, n .
$$

Let us introduce the "row-to-row" monodromy matrix $T(u)$, an $n \times n$ matrix with operatorvalued elements acting on $\mathbf{V}^{\otimes N}$,

$$
T_{0}(u)=R_{0 N}\left(u-\theta_{N}\right) R_{0 N-1}\left(u-\theta_{N-1}\right) \cdots R_{01}\left(u-\theta_{1}\right) .
$$

Here $\left\{\theta_{j} \mid j=1, \cdots, N\right\}$ are generic free complex parameters usually called as inhomogeneity parameters. The transfer matrix $t(u)$ of the associated spin chain with antiperiodic boundary condition (2.14) can be constructed similarly as [68]

$$
t(u)=\operatorname{tr}_{0}\left\{g_{0} T_{0}(u)\right\} .
$$

The QYBE and the relation (2.13) lead to the fact that the transfer matrices $t(u)$ given by (2.16) with different spectral parameters are mutually commuting: $[t(u), t(v)]=0$. The Hamiltonian (2.1) with the anti-periodic boundary condition (2.14) can be obtained from the transfer matrix as

$$
H=\left.\sinh \eta \frac{\partial \ln t(u)}{\partial u}\right|_{u=0,\left\{\theta_{j}\right\}=0} .
$$

The eigenvalues $\Lambda(u)$ of the transfer matrix $t(u)$ in case of $\mathrm{su}(3)$ are given in terms of an inhomogeneous $T-Q$ relation [59]

$$
\begin{aligned}
\Lambda(u)=e^{\frac{u}{3}}\{ & e^{\phi_{1}} e^{u} a(u) \frac{Q^{(1)}(u-\eta)}{Q^{(2)}(u)}+e^{-\phi_{1}} \omega e^{-u-\frac{2 \eta}{3}} d(u) \frac{Q^{(2)}(u+\eta) Q^{(3)}(u-\eta)}{Q^{(1)}(u) Q^{(4)}(u)} \\
& +\omega^{2} e^{-u-\frac{4 \eta}{3}} d(u) \frac{Q^{(4)}(u+\eta)}{Q^{(3)}(u)}+a(u) d(u) \frac{Q^{(3)}(u-\eta) f_{1}(u)}{Q^{(1)}(u) Q^{(2)}(u)} \\
& \left.+a(u) d(u) \frac{Q^{(2)}(u+\eta) f_{2}(u)}{Q^{(3)}(u) Q^{(4)}(u)}\right\},
\end{aligned}
$$


where

$$
\begin{aligned}
a(u) & =\prod_{l=1}^{N} \sinh \left(u-\theta_{l}+\eta\right), & d(u) & =\prod_{l=1}^{N} \sinh \left(u-\theta_{l}\right)=a(u-\eta), \\
Q^{(i)}(u) & =\prod_{l=1}^{N} \sinh \left(u-\lambda_{l}^{(i)}\right), & i & =1,2,3,4,
\end{aligned}
$$

$\omega=e^{\frac{2 i \pi}{3}}$ and the functions $f_{1}(u)$ and $f_{2}(u)$ are given by

$$
f_{1}(u)=f_{1}^{(+)} e^{u}+f_{1}^{(-)} e^{-u}, \quad f_{2}(u)=f_{2}^{(-)} e^{-u} .
$$

The $4 N+4$ parameters $\left\{\lambda_{l}^{(i)} \mid l=1, \cdots, N ; i=1,2,3,4\right\}, f_{1}^{( \pm)}, f_{2}^{(-)}$and $e^{\phi_{1}}$ satisfy the associated BAEs:

$$
\begin{aligned}
& \omega e^{-\phi_{1}} e^{-\lambda_{j}^{(1)}-\frac{2 \eta}{3}} \frac{Q^{(2)}\left(\lambda_{j}^{(1)}+\eta\right)}{Q^{(4)}\left(\lambda_{j}^{(1)}\right)}+a\left(\lambda_{j}^{(1)}\right) \frac{f_{1}\left(\lambda_{j}^{(1)}\right)}{Q^{(2)}\left(\lambda_{j}^{(1)}\right)}=0, \quad j=1, \cdots, N, \\
& e^{\phi_{1}} e^{\lambda_{j}^{(2)}} Q^{(1)}\left(\lambda_{j}^{(2)}-\eta\right)+d\left(\lambda_{j}^{(2)}\right) \frac{Q^{(3)}\left(\lambda_{j}^{(2)}-\eta\right) f_{1}\left(\lambda_{j}^{(2)}\right)}{Q^{(1)}\left(\lambda_{j}^{(2)}\right)}=0, \quad j=1, \cdots, N, \\
& \omega^{2} e^{-\lambda_{j}^{(3)}-\frac{4 \eta}{3}} Q^{(4)}\left(\lambda_{j}^{(3)}+\eta\right)+a\left(\lambda_{j}^{(3)}\right) \frac{Q^{(2)}\left(\lambda_{j}^{(3)}+\eta\right) f_{2}\left(\lambda_{j}^{(3)}\right)}{Q^{(4)}\left(\lambda_{j}^{(3)}\right)}=0, \quad j=1, \cdots, N, \\
& \omega e^{-\phi_{1}} e^{-\lambda_{j}^{(4)}-\frac{2 \eta}{3}} \frac{Q^{(3)}\left(\lambda_{j}^{(4)}-\eta\right)}{Q^{(1)}\left(\lambda_{j}^{(4)}\right)}+a\left(\lambda_{j}^{(4)}\right) \frac{f_{2}\left(\lambda_{j}^{(4)}\right)}{Q^{(3)}\left(\lambda_{j}^{(4)}\right)}=0, \quad j=1, \cdots, N, \\
& e^{\phi_{1}} e^{-\Theta-\chi^{(1)}+\chi^{(2)}}+e^{-2 \Theta+\chi^{(1)}+\chi^{(2)}-\chi^{(3)}} f_{1}^{(+)}=0, \\
& \omega e^{-\phi_{1}} e^{-\frac{2 \eta}{3}+\Theta-\chi^{(1)}+\chi^{(2)}+\chi^{(3)}-\chi^{(4)}}+\omega^{2} e^{-\frac{4 \eta}{3}+\Theta-\chi^{(3)}+\chi^{(4)}-N \eta} \\
& +e^{2 \Theta-N \eta}\left\{e^{-\chi^{(1)}-\chi^{(2)}+\chi^{(3)}+N \eta} f_{1}^{(-)}+e^{+\chi^{(2)}-\chi^{(3)}-\chi^{(4)}-N \eta} f_{2}^{(-)}\right\}=0, \\
& \omega e^{-\Theta-\chi^{(3)}+\chi^{(4)}}+\omega^{2} e^{\phi_{1}} e^{-\frac{2 \eta}{3}-\Theta-\chi^{(1)}+\chi^{(2)}+\chi^{(3)}-\chi^{(4)}+N \eta} \\
& +e^{-2 \Theta+N \eta}\left\{\omega^{2} e^{-\frac{2 \eta}{3}+\chi^{(1)}+\chi^{(2)}-\chi^{(4)}} f_{1}^{(+)}+e^{\phi_{1}} e^{\frac{2 \eta}{3}-\chi^{(1)}+\chi^{(3)}+\chi^{(4)}+N \eta} f_{2}^{(-)}\right\}=0, \\
& e^{-\phi_{1}} e^{-\frac{4 \eta}{3}+\Theta-\chi^{(1)}+\chi^{(2)}-N \eta}+\omega^{2} e^{-\frac{2 \eta}{3}+2 \Theta-\chi^{(1)}-\chi^{(2)}+\chi^{(4)}-N \eta} f_{1}^{(-)}=0,
\end{aligned}
$$

where

$$
\Theta=\sum_{l=1}^{N} \theta_{l}, \quad \chi^{(i)}=\sum_{l=1}^{N} \lambda_{l}^{(i)}, \quad i=1,2,3,4 .
$$

In homogeneous limit: $\left\{\theta_{j} \rightarrow 0\right\}$, the resulting $T-Q$ relation (2.18) and the associated BAEs (2.20)-(2.27) give rise to the eigenvalue and BAEs of the corresponding homogeneous spin chain (i.e., the $\mathrm{su}(3)$ spin torus). 


\section{$3 \quad$ Nested SoV basis}

In this section, we propose a convenient basis of the Hilbert space parameterized by the $N$ generic inhomogeneity parameters $\left\{\theta_{j} \mid j=1, \cdots, N\right\}$. It is found that actions of all the monodromy matrix elements on a basis vector take drastically simple forms like those in the so-called F-basis ${ }^{3}$ [69-73]. All these ingredients allow us to construct exact eigenstates of the $\operatorname{su}(n)$ spin torus model.

For convenience, let us introduce the notations

$$
\begin{array}{rlrl}
A(u) & =T_{1}^{1}(u), \quad B_{i}(u)=T_{i}^{1}(u), \quad C^{i}(u)=T_{1}^{i}(u), & \text { for } i & =2, \ldots, n, \\
D_{j}^{i}(u) & =T_{j}^{i}(u), & \text { for } i, j=2, \ldots, n .
\end{array}
$$

The exchange relations among the above operators are listed in appendix A. Let us introduce further the left quasi-vacuum state $\langle 0|$ and the right quasi-vacuum state $|0\rangle$

$$
\langle 0|=\langle 1, \cdots, 1|, \quad| 0\rangle=| 1,1, \cdots, 1\rangle
$$

The operators (3.1)-(3.2) acting on the states give rise to

$$
\begin{aligned}
\langle 0| A(u) & =a(u)\langle 0|, & & \langle 0| D_{i}^{l}(u)=d(u) \delta_{i}^{l}\langle 0|, & i, l & =2, \cdots, n, \\
\langle 0| B_{i}(u) & =0, & & \langle 0| C^{i}(u) \neq 0, & i & =2, \cdots, n, \\
A(u)|0\rangle & =a(u)|0\rangle, & & D_{i}^{l}(u)|0\rangle=d(u) \delta_{i}^{l}|0\rangle, & i, l & =2, \cdots, n, \\
C^{i}(u)|0\rangle & =0, & & B_{i}(u)|0\rangle \neq 0, & i & =2, \cdots, n,
\end{aligned}
$$

where the functions $a(u)$ and $d(u)$ are given by (2.19).

In the following part of this section, taking the su(3) spin chain as an example, we construct a nested SoV basis of the Hilbert space. The generalization to the $\mathrm{su}(n)$ case is given in appendix B. For two non-negative integers $m_{2}$ and $m$ such that $m_{2} \leq m \leq N$, let us introduce $m$ positive integers $P=\left\{p_{1}, \cdots, p_{m}\right\}$ such that

$$
1 \leq p_{1}<p_{2}<\cdots<p_{m_{2}} \leq N, \quad 1 \leq p_{m_{2}+1}<\cdots<p_{m} \leq N, \quad \text { and } \quad p_{j} \neq p_{l} .
$$

For each $P$ satisfies the above condition, let us introduce left and right states parameterized by the $N$ inhomogeneity parameters $\left\{\theta_{j}\right\}$ as follows:

$$
\begin{aligned}
\left\langle\theta_{p_{1}}, \cdots, \theta_{p_{m_{2}}} ; \theta_{p_{m_{2}+1}}, \cdots, \theta_{p_{m}}\right| & =\langle 0| C^{2}\left(\theta_{p_{1}}\right) \cdots C^{2}\left(\theta_{p_{m_{2}}}\right) C^{3}\left(\theta_{p_{m_{2}+1}}\right) \cdots C^{3}\left(\theta_{p_{m}}\right) \\
\left|\theta_{p_{1}}, \cdots, \theta_{p_{m_{2}}} ; \theta_{p_{m_{2}+1}}, \cdots, \theta_{p_{m}}\right\rangle & =B_{3}\left(\theta_{p_{m}}\right) \cdots B_{3}\left(\theta_{p_{m_{2}+1}}\right) B_{2}\left(\theta_{p_{m_{2}}}\right) \cdots B_{2}\left(\theta_{p_{1}}\right)|0\rangle
\end{aligned}
$$

where $m_{2}$ (resp. $m-m_{2}$ ) is the number of the operators $C^{2}(u)$ or $B_{2}(u)$ (resp. $C^{3}(u)$ or $\left.B_{3}(u)\right)$.

\footnotetext{
${ }^{3}$ It is interesting to study the relation between this basis and the F-basis [71, 73].
} 
It is easy to check that the states (3.9) and the states (3.10) are eigenstates of the operator $D_{3}^{3}(u)$, namely,

$$
\begin{aligned}
\left\langle\theta_{p_{1}}, \cdots, \theta_{p_{m_{2}}} ; \theta_{p_{m_{2}+1}}, \cdots, \theta_{p_{m}}\right| D_{3}^{3}(u)= & d(u) \prod_{l=m_{2}+1}^{m} \frac{\sinh \left(u-\theta_{p_{l}}+\eta\right)}{\sinh \left(u-\theta_{p_{l}}\right)} \\
& \times\left\langle\theta_{p_{1}}, \cdots, \theta_{p_{m_{2}}} ; \theta_{p_{m_{2}+1}}, \cdots, \theta_{p_{m}}\right|, \\
D_{3}^{3}(u)\left|\theta_{p_{1}}, \cdots, \theta_{p_{m_{2}}} ; \theta_{p_{m_{2}}+1}, \cdots, \theta_{p_{m}}\right\rangle= & d(u) \prod_{l=m_{2}+1}^{m} \frac{\sinh \left(u-\theta_{p_{l}}+\eta\right)}{\sinh \left(u-\theta_{p_{l}}\right)} \\
& \times\left|\theta_{p_{1}}, \cdots, \theta_{p_{m_{2}}} ; \theta_{p_{m_{2}+1}}, \cdots, \theta_{p_{m}}\right\rangle .
\end{aligned}
$$

Noting the fact that $d\left(\theta_{l}\right)=0, l=1, \cdots, N$ and using the exchange relations (A.1)-(A.10), we can derive some useful relations

$$
\begin{array}{ll}
\left\langle\theta_{p_{1}}, \cdots, \theta_{p_{m_{2}}} ; \theta_{p_{m_{2}}}, \cdots, \theta_{p_{m}}\right| D_{j}^{i}\left(\theta_{p_{l}}\right)=0, & l=m+1, \cdots, N, \text { and } i, j=2,3, \\
\left\langle\theta_{p_{1}}, \cdots, \theta_{p_{m_{2}}} ; \theta_{p_{m_{2}+1}}, \cdots, \theta_{p_{m}}\right| B_{i}\left(\theta_{p_{l}}\right)=0, & l=m+1, \cdots, N, \text { and } i=2,3, \\
D_{j}^{i}\left(\theta_{p_{l}}\right)\left|\theta_{p_{1}}, \cdots, \theta_{p_{m_{2}}} ; \theta_{p_{m_{2}+1}}, \cdots, \theta_{p_{m}}\right\rangle=0, & l=m+1, \cdots, N, \text { and } i=2,3, \\
C^{i}\left(\theta_{p_{l}}\right)\left|\theta_{p_{1}}, \cdots, \theta_{p_{m_{2}}} ; \theta_{p_{m_{2}+1}}, \cdots, \theta_{p_{m}}\right\rangle=0, & l=m+1, \cdots, N, \text { and } i=2,3 .
\end{array}
$$

The above relations and the exchange relations (A.1)-(A.10) allow us to derive the orthogonal relations between the left states and the right states

$$
\begin{array}{r}
\left\langle\theta_{p_{1}}, \cdots, \theta_{p_{m_{2}}} ; \theta_{p_{m_{2}+1}}, \cdots, \theta_{p_{m}} \mid \theta_{q_{1}}, \cdots, \theta_{q_{m_{2}}} ; \theta_{q_{m_{2}}+1}, \cdots, \theta_{q_{m^{\prime}}}\right\rangle= \\
\delta_{m, m^{\prime}} \delta_{m_{2}, m_{2}^{\prime}} \prod_{k=1}^{m} \delta_{p_{k}, q_{k}} G_{m}\left(\theta_{p_{1}}, \cdots, \theta_{p_{m_{2}}} \mid \theta_{p_{m_{2}+1}}, \cdots, \theta_{p_{m}}\right),
\end{array}
$$

where the factor $G_{m}\left(\theta_{p_{1}}, \cdots, \theta_{p_{m_{2}}} \mid \theta_{p_{m_{2}+1}}, \cdots, \theta_{p_{m}}\right)$ is given by

$$
\begin{aligned}
G_{m}\left(\theta_{p_{1}}, \cdots, \theta_{p_{m_{2}}} \mid \theta_{p_{m_{2}+1}}, \cdots, \theta_{p_{m}}\right)= & \\
& \prod_{k=1}^{m_{2}} \sinh \eta d_{p_{k}}\left(\theta_{p_{k}}\right) a\left(\theta_{p_{k}}\right) \prod_{l=1, l \neq k}^{m_{2}} \frac{\sinh \left(\theta_{p_{k}}-\theta_{p_{l}}+\eta\right)}{\sinh \left(\theta_{p_{k}}-\theta_{p_{l}}\right)} \\
& \times \prod_{k=m_{2}+1}^{m} \sinh \eta d_{p_{k}}\left(\theta_{p_{k}}\right) a\left(\theta_{p_{k}}\right)\left\{\prod_{l=m_{2}+1, l \neq k}^{m} \frac{\sinh \left(\theta_{p_{k}}-\theta_{p_{l}}+\eta\right)}{\sinh \left(\theta_{p_{k}}-\theta_{p_{l}}\right)}\right. \\
& \left.\times \prod_{l=1}^{m_{2}} \frac{\sinh \left(\theta_{p_{k}}-\theta_{p_{l}}-\eta\right)}{\sinh \left(\theta_{p_{k}}-\theta_{p_{l}}\right)}\right\} .
\end{aligned}
$$

Here the functions $\left\{d_{l}(u)\right\}$ are given by

$$
d_{l}(u)=\prod_{k=1, k \neq l}^{N} \sinh \left(u-\theta_{k}\right), \quad l=1, \cdots, N .
$$

On the other hand, we know that the total number of the linear-independent left (right) states given in $(3.9)((3.10))$ is

$$
\sum_{m=0}^{N} \frac{N !}{(N-m) ! m !} \sum_{m_{2}=0}^{m} \frac{m !}{\left(m-m_{2}\right) ! m_{2} !}=\sum_{m=0}^{N} \frac{N !}{(N-m) ! m !} 2^{m}=3^{N} .
$$


Thus these right (left) states form an orthogonal right (left) basis of the Hilbert space, namely,

$$
\begin{aligned}
\mathrm{id}= & \sum_{m=0}^{N} \sum_{m_{2}=0}^{m} \sum_{P} \frac{1}{G_{m}\left(\theta_{p_{1}}, \cdots, \theta_{p_{m_{2}}} \mid \theta_{p_{m_{2}+1}}, \cdots, \theta_{p_{m}}\right)} \\
& \times\left|\theta_{p_{1}}, \cdots, \theta_{p_{m_{2}}} ; \theta_{p_{m_{2}+1}}, \cdots, \theta_{p_{m}}\right\rangle\left\langle\theta_{p_{1}}, \cdots, \theta_{p_{m_{2}}} ; \theta_{p_{m_{2}+1}}, \cdots, \theta_{p_{m}}\right|
\end{aligned}
$$

where the notation $\sum_{P}$ indicates the sum over all $P$ satisfying the condition (3.8). Hence any right (left) state can be decomposed as a unique linear combination of these basis. Moreover, direct calculation shows that actions of the monodromy matrix elements on this basis become drastically simple (see below (3.22)-(3.26)). Here we list some of them relevant for us to construct eigenstates of the transfer matrix in the next section,

$$
\begin{aligned}
\left\langle\theta_{p_{1}}, \cdots,\right. & \theta_{p_{m_{2}}} ; \theta_{p_{m_{2}+1}}, \cdots, \theta_{p_{m}} \mid D_{3}^{3}(u)= \\
& d(u) \prod_{l=m_{2}+1}^{m} \frac{\sinh \left(u-\theta_{p_{l}}+\eta\right)}{\sinh \left(u-\theta_{p_{l}}\right)} \\
& \times\left\langle\theta_{p_{1}}, \cdots, \theta_{p_{m_{2}}} ; \theta_{p_{m_{2}+1}}, \cdots, \theta_{p_{m}}\right|, \\
\left\langle\theta_{p_{1}}, \cdots,\right. & \theta_{p_{m_{2}}} ; \theta_{p_{m_{2}+1}}, \cdots, \theta_{p_{m}} \mid D_{3}^{2}(u)= \\
& \sum_{l=m_{2}+1}^{m} \frac{\sinh \eta e^{\frac{u-\theta_{p_{l}}}{3}} d(u)}{\sinh \left(u-\theta_{p_{l}}\right)} \\
& \times \prod_{k=m_{2}+1, k \neq l}^{m} \frac{\sinh \left(u-\theta_{p_{k}}+\eta\right)}{\sinh \left(u-\theta_{p_{k}}\right)} \frac{\sinh \left(\theta_{p_{l}}-\theta_{p_{k}}-\eta\right)}{\sinh \left(\theta_{p_{l}}-\theta_{p_{k}}\right)} \\
& \times\left\langle\theta_{p_{1}}, \cdots, \theta_{p_{m_{2}}} ; \theta_{p_{m_{2}+1}}, \cdots, \theta_{p_{l-1}}, \theta_{p_{l+1}}, \cdots, \theta_{p_{m}}\right|, \\
\left\langle\theta_{p_{1}}, \cdots,\right. & \theta_{p_{m_{2}}} ; \theta_{p_{m_{2}+1}}, \cdots, \theta_{p_{m}} \mid D_{2}^{3}(u)= \\
& \sum_{l=1}^{m_{2}} \frac{\sinh \eta e^{-\frac{u-\theta_{p_{l}}}{3}} d(u)}{\sinh \left(u-\theta_{p_{l}}\right)} \\
& \times\left\{\prod_{k=1, k \neq l}^{m_{2}} \frac{\sinh \left(\theta_{p_{l}}-\theta_{p_{k}}+\eta\right)}{\sinh \left(\theta_{p_{l}}-\theta_{p_{k}}\right)} \prod_{k=m_{2}+1}^{m} \frac{\sinh \left(u-\theta_{p_{k}}+\eta\right)}{\sinh \left(u-\theta_{p_{k}}\right)}\right\} \\
& \times\left\langle\theta_{p_{1}}, \cdots, \theta_{p_{l-1}}, \theta_{p_{l+1}}, \cdots, \theta_{p_{m_{2}}} ; \theta_{p_{m_{2}+1}}, \cdots, \theta_{p_{m}}\right|,
\end{aligned}
$$




$$
\begin{aligned}
& \left\langle\theta_{p_{1}}, \cdots, \theta_{p_{m_{2}}} ; \theta_{p_{m_{2}+1}}, \cdots, \theta_{p_{m}}\right| B_{3}(u)= \\
& \sum_{l=m_{2}+1}^{m} \frac{\sinh \eta e^{-\frac{u-\theta_{p_{l}}}{3}} d(u)}{\sinh \left(u-\theta_{p_{l}}\right)} a\left(\theta_{p_{l}}\right) \\
& \times \prod_{k=m_{2}+1, k \neq l}^{m} \frac{\sinh \left(u-\theta_{p_{k}}+\eta\right)}{\sinh \left(u-\theta_{p_{k}}\right)} \frac{\sinh \left(\theta_{p_{l}}-\theta_{p_{k}}-\eta\right)}{\sinh \left(\theta_{p_{l}}-\theta_{p_{k}}\right)} \\
& \times \prod_{\alpha=1}^{m_{2}} \frac{\sinh \left(\theta_{p_{l}}-\theta_{p_{\alpha}}-\eta\right)}{\sinh \left(\theta_{p_{l}}-\theta_{p_{\alpha}}\right)}\left\langle\theta_{p_{1}}, \cdots, \theta_{p_{m_{2}}} ; \theta_{p_{m_{2}+1}}, \cdots, \theta_{p_{l-1}}, \theta_{p_{l+1}}, \cdots, \theta_{p_{m}}\right| \\
& +\sum_{l=m_{2}+1}^{m} \frac{\sinh \eta e^{-\frac{u-\theta_{p_{l}}}{3}} d(u)}{\sinh \left(u-\theta_{p_{l}}\right)} \prod_{k=m_{2}+1, k \neq l}^{m} \frac{\sinh \left(u-\theta_{p_{k}}+\eta\right)}{\sinh \left(u-\theta_{p_{k}}\right)} \frac{\sinh \left(\theta_{p_{l}}-\theta_{p_{k}}-\eta\right)}{\sinh \left(\theta_{p_{l}}-\theta_{p_{k}}\right)} \\
& \times \sum_{\alpha=1}^{m_{2}} \frac{\sinh \eta e^{-\frac{\theta_{p_{\alpha}}-\theta_{p_{l}}}{3}}}{\sinh \left(\theta_{p_{l}}-\theta_{p_{\alpha}}\right)} a\left(\theta_{p_{\alpha}}\right) \prod_{k=1, k \neq \alpha}^{m_{2}} \frac{\sinh \left(\theta_{p_{\alpha}}-\theta_{p_{k}}-\eta\right)}{\sinh \left(\theta_{p_{\alpha}}-\theta_{p_{k}}\right)} \\
& \times\left\langle\theta_{p_{1}}, \cdots, \theta_{p_{\alpha-1}}, \theta_{p_{l}}, \theta_{p_{\alpha+1}}, \cdots, \theta_{p_{m_{2}}} ; \theta_{p_{m_{2}+1}}, \cdots, \theta_{p_{l-1}}, \theta_{p_{l+1}}, \cdots, \theta_{p_{m}}\right|, \\
& \left\langle\theta_{p_{1}}, \cdots, \theta_{p_{m_{2}}} ; \theta_{p_{m_{2}+1}}, \cdots, \theta_{p_{m}}\right| C^{3}(u)= \\
& \sum_{l=m+1}^{N} \frac{e^{\frac{u-\theta_{p_{l}}}{3}}}{\sinh \left(u-\theta_{p_{l}}\right)} \frac{d(u)}{d_{p_{l}}\left(\theta_{p_{l}}\right)} \\
& \times \prod_{k=m_{2}+1}^{m} \frac{\sinh \left(u-\theta_{p_{k}}+\eta\right)}{\sinh \left(u-\theta_{p_{k}}\right)} \frac{\sinh \left(\theta_{p_{l}}-\theta_{p_{k}}\right)}{\sinh \left(\theta_{p_{l}}-\theta_{p_{k}}+\eta\right)} \\
& \times\left\langle\theta_{p_{1}}, \cdots \theta_{p_{m_{2}}} ; \theta_{p_{m_{2}+1}}, \cdots, \theta_{p_{m}}, \theta_{p_{l}}\right| \\
& +\sum_{l=m+1}^{N} \sum_{\alpha=1}^{m_{2}} \frac{e^{\frac{u-\theta_{p_{\alpha}}}{3}}}{\sinh \left(u-\theta_{p_{\alpha}}\right)} \prod_{k=m_{2}+1}^{m} \frac{\sinh \left(u-\theta_{p_{k}}+\eta\right)}{\sinh \left(u-\theta_{p_{k}}\right)} \frac{\sinh \left(\theta_{p_{l}}-\theta_{p_{k}}\right)}{\sinh \left(\theta_{p_{l}}-\theta_{p_{k}}+\eta\right)} \\
& \times \frac{\sinh \eta d(u) e^{\frac{\theta_{p_{l}}-\theta_{p_{\alpha}}}{3}}}{d_{p_{l}}\left(\theta_{p_{l}}\right) \sinh \left(\theta_{p_{\alpha}}-\theta_{p_{l}}-\eta\right)} \prod_{k=1, k \neq \alpha}^{m_{2}} \frac{\sinh \left(\theta_{p_{l}}-\theta_{p_{k}}\right)}{\sinh \left(\theta_{p_{l}}-\theta_{p_{k}}+\eta\right)} \frac{\sinh \left(\theta_{p_{\alpha}}-\theta_{p_{k}}+\eta\right)}{\sinh \left(\theta_{p_{\alpha}}-\theta_{p_{k}}\right)} \\
& \times\left\langle\theta_{p_{1}}, \cdots, \theta_{p_{\alpha-1}}, \theta_{p_{l}}, \theta_{p_{\alpha+1}}, \cdots, \theta_{p_{m_{2}}} ; \theta_{p_{m_{2}+1}}, \cdots, \theta_{p_{m}}, \theta_{p_{\alpha}}\right| .
\end{aligned}
$$

The sketch proof of the above operator decompositions is given in appendix C. Similarly, one may derive operator decompositions on the right basis which also have simple forms as $(3.22)-(3.26)$.

Some remarks are in order. In the rational limit, ${ }^{4}$ the resulting basis serves as the SoV basis for the associated rational spin chain model. ${ }^{5}$ We have checked that each basis vector given by (3.9) and (3.10) for the $\mathrm{su}(3)$ case (the generalizations to the $\mathrm{su}(n)$ case are given in appendix B, see (B.1) and (B.2) below ) is an off-shell Bethe state obtained via the nested algebraic Bethe Ansatz [75, 76] by replacing the Bethe roots with some sets

\footnotetext{
${ }^{4}$ Redefine: $u \rightarrow \epsilon u, \theta_{j} \rightarrow \epsilon \theta_{j}$ and $\eta \rightarrow \epsilon \eta$, then take the limit $\epsilon \rightarrow 0$.

${ }^{5}$ The resulting SoV basis for the rational spin chain model is different from that in [74]. It is interesting to study the relation between them.
} 
of the inhomogeneity parameters. ${ }^{6}$ This observation provides an efficient way to construct similar nested SoV basis for general high-rank quantum integrable models. From explicit expressions (3.22)-(3.26), one can see that in the basis (3.9) the operators have no compensating exchange terms on the level of the local operators (i.e. polarization free), which have similar simple forms as those in the F-basis [70-73] and allow us to compute correlation functions [77] for quantum spin chains associated with higher-rank algebras [78-81].

\section{Eigenstates of the transfer matrix}

In this section, we adopt the method developed in [37, 38] (see also [30]) to construct eigenstates of the $\mathrm{su}(3)$ spin torus based on the inhomogeneous $T-Q$ relations given by (2.18) [59] and the basis introduced in the previous section. For the su(3) case, the monodromy matrix is expressed in terms of the operators (3.1)-(3.2) as

$$
T(u)=\left(\begin{array}{ccc}
A(u) & B_{2}(u) & B_{3}(u) \\
C^{2}(u) & D_{2}^{2}(u) & D_{3}^{2}(u) \\
C^{3}(u) & D_{2}^{3}(u) & D_{3}^{3}(u)
\end{array}\right) .
$$

The corresponding transfer matrix (2.16) reads

$$
t(u)=B_{2}(u)+D_{3}^{2}(u)+C^{3}(u) .
$$

The commutativity of the transfer matrices $t(u)$ with different spectral parameters implies that they have common eigenstates. Let $|\Psi\rangle$ be a common eigenstate of $t(u)$, which does not depend upon $u$, with an eigenvalue $\Lambda(u)$, i.e.,

$$
t(u)|\Psi\rangle=\Lambda(u)|\Psi\rangle
$$

where the eigenvalue $\Lambda(u)$ of the transfer matrix $t(u)$ is given by the inhomogeneous $T-$ $Q$ relation (2.18). Due to the fact that the left states $\left\{\left\langle\theta_{p_{1}}, \cdots, \theta_{p_{m_{2}}} ; \theta_{p_{m_{2}+1}}, \cdots, \theta_{p_{m}}\right|\right.$, $\left.m_{2}=0, \cdots, m ; m=0, \cdots, N\right\}$ given by (3.9) form a basis of the dual Hilbert space, the eigenstate $|\Psi\rangle$ is completely determined (up to an overall scalar factor) by the following scalar products $[29,37,38]$

$$
\begin{gathered}
F_{m_{2}, m-m_{2}}\left(\theta_{p_{1}}, \cdots, \theta_{p_{m_{2}}} ; \theta_{p_{m_{2}+1}}, \cdots, \theta_{p_{m}}\right)=\left\langle\theta_{p_{1}}, \cdots, \theta_{p_{m_{2}}} ; \theta_{p_{m_{2}+1}}, \cdots, \theta_{p_{m}} \mid \Psi\right\rangle, \\
1 \leq p_{1}<\cdots<p_{m_{2}}, \quad 1 \leq p_{m_{2}+1}<\cdots<p_{m} \leq N, \quad p_{j} \neq p_{k}, \quad 0 \leq m_{2} \leq m \leq N .
\end{gathered}
$$

\footnotetext{
${ }^{6} \mathrm{~A}$ general off-shell Bethe state is $\overline{\left|\lambda_{1}, \cdots, \lambda_{m} ; \lambda_{1}^{(1)}, \cdots, \lambda_{m-m_{2}}^{(1)}\right\rangle}=B_{i_{1}}\left(\lambda_{1}\right) \cdots B_{i_{m}}\left(\lambda_{m}\right) F^{i_{1}, \cdots, i_{m}}|0\rangle$, where $\left\{F^{i_{1}, \cdots, i_{m}} \mid i_{l}=2,3\right\}$ are the vector components of a nested off-shell Bethe state $B^{(1)}\left(\lambda_{1}^{(1)}\right) \cdots B^{(1)}\left(\lambda_{m-m_{2}}^{(1)}\right)|0\rangle^{(1)}=\sum_{i_{1}, \cdots, i_{m}=2}^{3} F^{i_{1}, \cdots, i_{m}}\left|i_{1}, \cdots, i_{m}\right\rangle^{(1)}$, and the operator $B^{(1)}(u)$ and $|0\rangle^{(1)}$ are the corresponding creation operator and the reference state associated with the nested $\operatorname{su}(2)$ spin chain with $m$ sites and the corresponding inhomogeneous parameters being $\left\{\lambda_{1}, \cdots, \lambda_{m}\right\}[75,76]$. For general values of $\lambda_{1}, \cdots, \lambda_{m}$ and $\lambda_{1}^{(1)}, \cdots, \lambda_{m-m_{2}}^{(1)}$, the Bethe sate $\overline{\left|\lambda_{1}, \cdots, \lambda_{m} ; \lambda_{1}^{(1)}, \cdots, \lambda_{m-m_{2}}^{(1)}\right\rangle}$ is a linear combination of the vectors (3.10) [80,81]. However, if the parameters $\left\{\lambda_{l} \mid l=1, \cdots, m\right\}$ are particularly chosen as $\left\{\lambda_{l}=\theta_{p_{l}} \mid l=1, \cdots, m\right\}$ and then the nested parameters $\left\{\lambda_{n}^{(1)} \mid n=1, \cdots, m-m_{2}\right\}$ have to take the values in the chosen set of $\left\{\lambda_{l} \mid l=1, \cdots, m\right\}$ (e.g., $\left\{\lambda_{n}^{(1)}=\theta_{p_{n}} \mid n=m_{2}+1, \cdots, m\right\}$ ), the corresponding linear combination becomes drastically simple such that only one term such as (3.10) does remain.
} 
Following [37, 38], let us consider the quantities $\left\langle\theta_{p_{1}}, \cdots, \theta_{p_{m_{2}}} ; \theta_{p_{m_{2}+1}}, \cdots, \theta_{p_{m}}\left|t\left(\theta_{p_{m+1}}\right)\right| \Psi\right\rangle$. Acting $t\left(\theta_{p_{m+1}}\right)$ to the right gives rise to the relation

$$
\begin{array}{r}
\Lambda\left(\theta_{p_{m+1}}\right) \\
F_{m_{2}, m-m_{2}}\left(\theta_{p_{1}}, \cdots, \theta_{p_{m_{2}}} ; \theta_{p_{m_{2}+1}}, \cdots, \theta_{p_{m}}\right)= \\
\left\langle\theta_{p_{1}}, \cdots, \theta_{p_{m_{2}}} ; \theta_{p_{m_{2}+1}}, \cdots, \theta_{p_{m}}\left|t\left(\theta_{p_{m+1}}\right)\right| \Psi\right\rangle .
\end{array}
$$

With the help of the expression (4.2) of the transfer matrix and the relations (3.13)-(3.14), by acting $t\left(\theta_{p_{m+1}}\right)$ to the left we readily obtain

$$
F_{m_{2}, m-m_{2}}\left(\theta_{p_{1}}, \cdots, \theta_{p_{m_{2}}} ; \theta_{p_{m_{2}+1}}, \cdots, \theta_{p_{m}}\right)=\left\{\prod_{l=m_{2}+1}^{m} \Lambda\left(\theta_{p_{l}}\right)\right\} F_{m_{2}}\left(\theta_{p_{1}}, \cdots, \theta_{p_{m_{2}}}\right),
$$

where the scalar products $F_{m}\left(\theta_{p_{1}}, \cdots, \theta_{p_{m}}\right)$ are given by

$$
F_{m}\left(\theta_{p_{1}}, \cdots, \theta_{p_{m}}\right)=\left\langle 0\left|C^{2}\left(\theta_{p_{1}}\right) \cdots C^{2}\left(\theta_{p_{m}}\right)\right| \Psi\right\rangle, \quad m=0, \cdots, N .
$$

It follows that in order to obtain all the scalar products (4.3) it is sufficient to compute the scalar products (4.6). After a tedious calculation, we have

$$
\begin{aligned}
F_{m}\left(\theta_{p_{1}}, \cdots, \theta_{p_{m}}\right)= & \sum_{1 \leq p_{1}^{\prime}<\cdots<p_{m}^{\prime} \leq N} g_{m}\left(\theta_{p_{1}}, \cdots, \theta_{p_{m}} \mid \theta_{p_{1}^{\prime}}, \cdots, \theta_{p_{m}^{\prime}}\right) \\
& \times \prod_{\alpha=1}^{m} \prod_{k=m+1}^{N} \sinh \left(\theta_{p_{\alpha}^{\prime}}-\theta_{p_{k}}+\eta\right) \frac{\prod_{l=1}^{m} \Lambda\left(\theta_{p_{l}^{\prime}}\right)}{f_{m}\left(\theta_{p_{1}^{\prime}}, \cdots, \theta_{p_{m}^{\prime}}\right)} \frac{\prod_{l=1}^{N} a\left(\theta_{k}\right)}{\prod_{k=m+1}^{N} \Lambda\left(\theta_{p_{k}}\right)}\langle\overline{0} \mid \Psi\rangle,
\end{aligned}
$$

where the state $\langle\overline{0}|=\langle 3, \cdots, 3|$ and the functions $g_{m}\left(v_{1}, \cdots, v_{m} \mid u_{1}, \cdots, u_{m}\right)$ and $f_{m}\left(\theta_{p_{1}}\right.$, $\left.\cdots, \theta_{p_{m}}\right)$ are given by

$$
\begin{aligned}
g_{m}\left(v_{1}, \cdots, v_{m} \mid u_{1}, \cdots, u_{m}\right) & =\frac{\prod_{\alpha=1}^{m} \prod_{k=1}^{m} \sinh \left(u_{\alpha}-v_{k}+\eta\right) \sinh \left(u_{\alpha}-v_{k}\right)}{\prod_{k<l}^{m} \sinh \left(u_{l}-u_{k}\right) \sinh \left(v_{k}-v_{l}\right)} \operatorname{det} \mathcal{M}, \\
f_{m}\left(\theta_{p_{1}}, \cdots, \theta_{p_{m}}\right) & =\prod_{l=1}^{m} \sinh \eta d_{p_{l}}\left(\theta_{p_{l}}\right) a\left(\theta_{p_{l}}\right) \prod_{k=1, k \neq l}^{m} \frac{\sinh \left(\theta_{p_{l}}-\theta_{p_{k}}+\eta\right)}{\sinh \left(\theta_{p_{l}}-\theta_{p_{k}}\right)}
\end{aligned}
$$

and $\mathcal{M}$ is an $m \times m$ matrix with matrix elements

$$
\mathcal{M}_{\alpha, k}=\frac{\sinh \eta e^{-\frac{u_{\alpha}-v_{k}}{3}}}{\sinh \left(u_{\alpha}-v_{k}+\eta\right) \sinh \left(u_{\alpha}-v_{k}\right)}, \quad \alpha, k=1, \cdots, m .
$$

The proof of (4.7) is given in appendix D.

The identity decomposition (3.21) allows us to retrieve the eigenstate $|\Psi\rangle$ of the transfer matrix corresponding to an eigenvalue $\Lambda(u)$ as

$$
\begin{aligned}
|\Psi\rangle= & \sum_{m=0}^{N} \sum_{m_{2}=0}^{m} \sum_{P} \frac{\left\langle\theta_{p_{1}}, \cdots, \theta_{p_{m_{2}}} ; \theta_{p_{m_{2}+1}}, \cdots, \theta_{p_{m}} \mid \Psi\right\rangle}{G_{m}\left(\theta_{p_{1}}, \cdots, \theta_{p_{m_{2}}} \mid \theta_{p_{m_{2}+1}}, \cdots, \theta_{p_{m}}\right)} \\
& \times\left|\theta_{p_{1}}, \cdots, \theta_{p_{m_{2}}} ; \theta_{p_{m_{2}+1}}, \cdots, \theta_{p_{m}}\right\rangle \\
= & \sum_{m=0}^{N} \sum_{m_{2}=0}^{m} \sum_{P} \frac{F_{m_{2}}\left(\theta_{p_{1}}, \cdots, \theta_{p_{m_{2}}}\right) \prod_{k=m_{2}+1}^{m} \Lambda\left(\theta_{p_{k}}\right)}{G_{m}\left(\theta_{p_{1}}, \cdots, \theta_{p_{m_{2}}} \mid \theta_{p_{m_{2}+1}}, \cdots, \theta_{p_{m}}\right)} \\
& \times\left|\theta_{p_{1}}, \cdots, \theta_{p_{m_{2}}} ; \theta_{p_{m_{2}}+1}, \cdots, \theta_{p_{m}}\right\rangle
\end{aligned}
$$


where the factors $F_{m_{2}}\left(\theta_{p_{1}}, \cdots, \theta_{p_{m_{2}}}\right)$ and $G_{m}\left(\theta_{p_{1}}, \cdots, \theta_{p_{m_{2}}} \mid \theta_{p_{m_{2}+1}}, \cdots, \theta_{p_{m}}\right)$ are given respectively by (4.7) and (3.18). It should be emphasized that the factor $F_{m_{2}}\left(\theta_{p_{1}}, \cdots, \theta_{p_{m_{2}}}\right)$ does depend upon the corresponding eigenvalue $\Lambda(u)$ associated with the eigenstate $|\Psi\rangle$, while $G_{m}\left(\theta_{p_{1}}, \cdots, \theta_{p_{m_{2}}} \mid \theta_{p_{m_{2}+1}}, \cdots, \theta_{p_{m}}\right)$ does not.

Some remarks are in order. In the homogeneous limit, the resulting eigenstate (4.11) (if it exists) becomes the eigenstate of the homogeneous quantum spin chain (i.e. the $\mathrm{su}(3)$ spin torus) due to the fact the $T-Q$ relation (2.18) and the associated BAEs (2.20)-(2.27) have well-defined homogeneous limits. We have checked that such a limit of the state (4.11) does exist for some small $N$. For an example, here we present the limit of the $N=2$ case

$$
\begin{gathered}
\lim _{\theta_{1}, \theta_{2} \rightarrow 0}|\Psi\rangle \propto|0\rangle+\frac{1}{\sinh ^{3} \eta}\left[\Lambda^{\prime} B_{3}+\Lambda B_{3}^{\prime}-2 \operatorname{coth} \eta \Lambda B_{3}\right]|0\rangle+\frac{\Lambda^{2}}{\sinh ^{8} \eta} B_{3} B_{3}|0\rangle \\
+\frac{\Lambda^{2}}{a^{2}(0)}\left\{\left[\left(\frac{8}{9}-2 \operatorname{coth} \eta \frac{\Lambda^{\prime}}{\Lambda}+\left(\frac{\Lambda^{\prime}}{\Lambda}\right)^{2}\right) B_{2}+\left(\frac{\Lambda^{\prime}}{\Lambda}-\operatorname{coth} \eta-\frac{1}{3}\right) B_{2}^{\prime}\right]\right. \\
+\frac{\Lambda}{\sinh ^{4} \eta}\left[\left(\operatorname{coth} \eta \frac{\Lambda^{\prime}}{\Lambda}-\frac{\Lambda^{\prime}}{3 \Lambda}-\frac{8}{9}\right) B_{3} B_{2}\right. \\
\left.\quad+\left(\frac{\Lambda^{\prime}}{\Lambda}-\operatorname{coth} \eta-\frac{1}{3}\right)\left(B_{3}^{\prime} B_{2}-B_{3} B_{2}^{\prime}\right)\right] \\
\left.+\frac{\Lambda^{2}}{\sinh ^{8} \eta} B_{2} B_{2}\right\}|0\rangle,
\end{gathered}
$$

where

$$
\begin{aligned}
B_{i} & =B_{i}(0), & B_{i}^{\prime} & =\left.\frac{\partial}{\partial u} B_{i}(u)\right|_{u=0}, \quad i=2,3, \\
\Lambda & =\Lambda(0), & \Lambda^{\prime} & =\left.\frac{\partial}{\partial u} \Lambda(u)\right|_{u=0} .
\end{aligned}
$$

It is conjectured that the eigenstate (4.11) for generic $N$ has a well-defined homogeneous limit. However, the direct proof remains an important open problem.

\section{Conclusions}

In this paper, we introduced a convenient basis of the Hilbert space, which could be treated as the SoV basis for the trigonometric spin chain model associated with the su(3) algebra. We have demonstrated that the monodromy matrix elements acting on a generic basis vector take simple forms such as (3.22)-(3.26) without compensating exchange terms on the level of the local operators (i.e. polarization free). With the help of this basis, the corresponding eigenstates of the transfer matrix can be constructed by (4.11) via its ODBA solution [59]. In the rational limit, the resulting basis serves as the SoV basis for the associated rational spin chain model. Moreover, as each basis vector is an off-shell Bethe state with the Bethe roots replaced by the inhomogeneous parameters, this procedure provides an efficient way to construct nested SoV basis for generic high-rank quantum integrable models such as the one-dimensional Hubbard model [82] with algebraic Bethe Ansatz. 


\section{Acknowledgments}

The financial supports from the National Natural Science Foundation of China (Grant Nos. 11375141, 11374334, 11434013, 11425522 and 11547045), BCMIIS and the Strategic Priority Research Program of the Chinese Academy of Sciences are gratefully acknowledged.

\section{A Exchange relations}

The QYBE implies the following exchange relations among the monodromy matrix elements

$$
\begin{aligned}
C^{l}(v) D_{i}^{k}(u) & =\sum_{\alpha, \beta=2}^{n} \frac{R_{\alpha \beta}^{k l}(u-v)}{\sinh (u-v)} D_{i}^{\alpha}(u) C^{\beta}(v)-\frac{R_{i 1}^{1 i}(u-v)}{\sinh (u-v)} D_{i}^{l}(v) C^{k}(u) \\
C^{k}(v) A(u) & =\frac{\sinh (u-v-\eta)}{\sinh (u-v)} A(u) C^{k}(v)+\frac{R_{1 k}^{k 1}(v-u)}{\sinh (u-v)} A(v) C^{k}(u) \\
{\left[C^{i}(u), B_{l}(v)\right]=} & \frac{1}{\sinh (u-v)}\left(R_{1 l}^{l 1}(u-v) A(v) D_{l}^{i}(u)-R_{1 i}^{i 1}(u-v) A(u) D_{l}^{i}(v)\right) \\
= & \frac{1}{\sinh (u-v)}\left(R_{l 1}^{1 l}(v-u) D_{l}^{i}(u) A(v)-R_{i 1}^{1 i}(v-u) D_{l}^{i}(v) A(u)\right) \\
A(u) B_{i}(v)= & \frac{\sinh (u-v-\eta)}{\sinh (u-v)} B_{i}(v) A(u)+\frac{R_{i 1}^{1 i}(v-u)}{\sinh (u-v)} B_{i}(u) A(v) \\
D_{i}^{j}(u) B_{l}(v)= & \sum_{\alpha, \beta=2}^{n} \frac{R_{i l}^{\alpha \beta}(u-v)}{\sinh (u-v)} B_{\beta}(v) D_{\alpha}^{j}(u)-\frac{R_{1 j}^{j 1}(u-v)}{\sinh (u-v)} B_{i}(u) D_{l}^{j}(v) \\
B_{i}(u) B_{j}(v)= & \sum_{\alpha, \beta=2}^{n} \frac{R_{i j}^{\alpha \beta}(u-v)}{\sinh (u-v+\eta)} B_{\beta}(v) B_{\alpha}(u) \\
C^{j}(v) C^{i}(u)= & \sum_{\alpha, \beta=2}^{n} \frac{R_{\alpha \beta}^{i j}(u-v)}{\sinh (u-v+\eta)} C^{\alpha}(u) C^{\beta}(v) \\
{\left[T_{\beta}^{\alpha}(u), T_{\beta}^{\alpha}(v)\right]=} & 0, \quad \alpha, \beta=1, \cdots, n, \\
{\left[T_{\alpha}^{\alpha}(u), T_{\beta}^{\beta}(v)\right]=} & \frac{1}{\sinh (u-v)}\left\{R_{\alpha \beta}^{\beta \alpha}(u-v) T_{\alpha}^{\beta}(v) T_{\beta}^{\alpha}(u)-R_{\beta \alpha}^{\alpha \beta}(u-v) T_{\alpha}^{\beta}(u) T_{\beta}^{\alpha}(v)\right\} \\
& \alpha \neq \beta, \quad \text { and } \quad \alpha, \beta=1, \cdots, n \\
{\left[T_{\beta}^{\alpha}(u), T_{\alpha}^{\beta}(v)\right]=} & \frac{R_{\beta \alpha}^{\alpha \beta}(u-v)}{\sinh (u-v)}\left\{T_{\beta}^{\beta}(v) T_{\alpha}^{\alpha}(u)-T_{\beta}^{\beta}(u) T_{\alpha}^{\alpha}(v)\right\} \\
& \alpha \neq \beta, \quad \operatorname{and} \quad \alpha, \beta=1, \cdots, n
\end{aligned}
$$




\section{B $\operatorname{su}(n)$ case}

For the $\operatorname{su}(n)$ spin chain, let us introduce $n-1$ non-negative integers $m_{2}, m_{3}, \cdots, m_{n}$ such that $\sum_{l=2}^{n} m_{l} \leq N$ and

$$
\begin{aligned}
& \left\langle\theta_{p_{1}}, \cdots, \theta_{p_{m_{2}}} ; \cdots ; \theta_{p_{m_{2}+\cdots m_{n-1}+1}}, \cdots, \theta_{p_{m_{2}+\cdots m_{n}}}\right|= \\
& \langle 0| C^{2}\left(\theta_{p_{1}}\right) \cdots C^{2}\left(\theta_{p_{m_{2}}}\right) \cdots C^{n}\left(\theta_{p_{m_{2}+\cdots+m_{n-1}+1}}\right) \cdots C^{n}\left(\theta_{p_{m_{2}+\cdots+m_{n}}}\right) \\
& \left|\theta_{p_{1}}, \cdots, \theta_{p_{m_{2}}} ; \cdots ; \theta_{p_{m_{2}+\cdots m_{n-1}+1}}, \cdots, \theta_{p_{m_{2}+\cdots m_{n}}}\right\rangle= \\
& \quad B_{n}\left(\theta_{p_{m_{2}+\cdots+m_{n}}}\right) \cdots B_{n}\left(\theta_{p_{m_{2}+\cdots+m_{n-1}+1}}\right) \cdots B_{2}\left(\theta_{p_{m_{2}}}\right) \cdots B_{2}\left(\theta_{p_{1}}\right)|0\rangle
\end{aligned}
$$

where $1 \leq p_{1}<\cdots<p_{m_{2}} \leq N, \cdots, 1 \leq p_{m_{2}+\cdots+m_{n-1}+1}<\cdots<p_{m_{2}+\cdots+m_{n}} \leq N$ and $p_{j} \neq p_{k}$. Note that the number of the operators $C^{j}(u)$ (or $\left.B_{j}(u)\right)$ in the above expression is $m_{j}$. Due to the fact that $d\left(\theta_{j}\right)=0$, with the help of the exchange relations (A.1) and (A.5), we can show that these states are in fact eigenstates of the operator $D_{n}^{n}(u)$

$$
\begin{aligned}
\left\langle\theta_{p_{1}}, \cdots, \theta_{p_{m_{2}}} ; \cdots ; \theta_{p_{m_{2}+\cdots m_{n-1}+1}}, \cdots\right. & , \theta_{p_{m_{2}+\cdots m_{n}}} \mid D_{n}^{n}(u)= \\
& d(u) \prod_{k=m_{2}+\cdots m_{n-1}+1}^{m_{2}+\cdots m_{n}} \frac{\sinh \left(u-\theta_{p_{k}}+\eta\right)}{\sinh \left(u-\theta_{p_{k}}\right)} \\
& \times\left\langle\theta_{p_{1}}, \cdots, \theta_{p_{m_{2}}} ; \cdots ; \theta_{p_{m_{2}+\cdots m_{n-1}+1}}, \cdots, \theta_{p_{m_{2}+\cdots m_{n}}}\right|,
\end{aligned}
$$

$$
\begin{aligned}
& D_{n}^{n}(u)\left|\theta_{p_{1}}, \cdots, \theta_{p_{m_{2}}} ; \cdots ; \theta_{p_{m_{2}+\cdots m_{n-1}+1}}, \cdots, \theta_{p_{m_{2}+\cdots m_{n}}}\right\rangle= \\
& d(u) \prod_{k=m_{2}+\cdots m_{n-1}+1}^{m_{2}+\cdots m_{n}} \frac{\sinh \left(u-\theta_{p_{k}}+\eta\right)}{\sinh \left(u-\theta_{p_{k}}\right)} \\
& \times\left|\theta_{p_{1}}, \cdots, \theta_{p_{m_{2}}} ; \cdots ; \theta_{p_{m_{2}+\cdots m_{n-1}+1}}, \cdots, \theta_{p_{m_{2}+\cdots m_{n}}}\right\rangle .
\end{aligned}
$$

For generic values of $\left\{\theta_{j}\right\}$, these right (left) states form an orthogonal right (left) basis of the Hilbert space, and any right (left) state can be decomposed as a unique linear combination of these basis.

Using the similar method in appendix $\mathrm{C}$, we can obtain the explicit expressions for the operators $\left\{D_{i}^{n}(u), D_{n}^{i}(u) \mid i=2, \cdots, n\right\}, B_{n}(u)$ and $C^{n}(u)$ in the basis (B.1). Like (3.22)(3.26), the operators take some simple forms without compensating exchange terms on the level of the local operators (i.e. polarization free) and hence have similar simple forms as those in the F-basis [70, 71, 73]. These resulting simple forms allow one to construct eigenstates of the transfer matrix of the $\mathrm{su}(n)$ spin torus via its ODBA solution [59].

\section{Proof of the operator decomposition}

Keeping the relations (3.11) and (3.13)-(3.14) in mind and using the exchange relations (A.1)-(A.10), one can easily check the actions (3.22)-(3.25) straightforwardly. In 
order to prove (3.26), we apply the identity decomposition (3.21) to the l.h.s. of (3.26), giving rise to

$$
\begin{aligned}
& \left\langle\theta_{p_{1}}, \cdots, \theta_{p_{m_{2}}} ; \theta_{p_{m_{2}+1}}, \cdots, \theta_{p_{m}}\right| C^{3}(u)= \\
& \quad \sum_{P^{\prime}}\left|\theta_{p_{1}^{\prime}}, \cdots, \theta_{p_{m_{2}}^{\prime}} ; \theta_{p_{m_{2}+1}^{\prime}}, \cdots, \theta_{p_{m+1}^{\prime}}\right\rangle \\
& \quad \times \frac{\left\langle\theta_{p_{1}}, \cdots, \theta_{p_{m_{2}}} ; \theta_{p_{m_{2}+1}}, \cdots, \theta_{p_{m}}\left|C^{3}(u)\right| \theta_{p_{1}^{\prime}}, \cdots, \theta_{p_{m_{2}}^{\prime}} ; \theta_{p_{m_{2}+1}^{\prime}}, \cdots, \theta_{p_{m+1}^{\prime}}\right\rangle}{G_{m+1}\left(\theta_{p_{1}^{\prime}}, \cdots, \theta_{p_{m_{2}}^{\prime}} \mid \theta_{p_{m_{2}+1}^{\prime}}, \cdots, \theta_{p_{m+1}^{\prime}}\right)},
\end{aligned}
$$

where the sum is over $P^{\prime}=\left\{p_{1}^{\prime}, \cdots, p_{m+1}^{\prime}\right\}$ such that $1 \leq p_{1}^{\prime}<\cdots<p_{m_{2}}^{\prime} \leq N, 1 \leq p_{m 2+1}^{\prime}<$ $\cdots<p_{m+1}^{\prime} \leq N$ and $p_{j}^{\prime} \neq p_{k}^{\prime}$. Since that the factor $G_{m+1}\left(\theta_{p_{1}^{\prime}}, \cdots, \theta_{p_{m_{2}}^{\prime}} \mid \theta_{p_{m_{2}+1}^{\prime}}, \cdots, \theta_{p_{m+1}^{\prime}}\right)$ is already known (3.18), it is sufficient to compute the scalar products

$$
\begin{aligned}
\left\langle\theta_{p_{1}}, \cdots,\right. & \left.\theta_{p_{m_{2}}} ; \theta_{p_{m_{2}+1}}, \cdots, \theta_{p_{m}}\left|C^{3}(u)\right| \theta_{p_{1}^{\prime}}, \cdots, \theta_{p_{m_{2}}^{\prime}} ; \theta_{p_{m_{2}+1}^{\prime}}, \cdots, \theta_{p_{m+1}^{\prime}}\right\rangle= \\
& \left\langle\theta_{p_{1}}, \cdots, \theta_{p_{m_{2}}} ; \theta_{p_{m_{2}+1}}, \cdots, \theta_{p_{m}}\right| C^{3}(u) B_{3}\left(\theta_{p_{m+1}^{\prime}}\right) \cdots B_{3}\left(\theta_{p_{m_{2}+1}^{\prime}}\right) \\
& \times B_{2}\left(\theta_{p_{m_{2}}^{\prime}}\right) \cdots B_{2}\left(\theta_{p_{1}^{\prime}}\right)|0\rangle .
\end{aligned}
$$

The relations (3.12), (3.15)-(3.16) and the exchange relations (A.1)-(A.10) allow us to arrive at the operator decomposition (3.26) by direct calculation. Similarly, we can work out the explicit decomposition expressions for the operators $D_{2}^{2}(u), B_{2}(u), C^{2}(u)$ and $A(u)$.

\section{Proof of (4.7)}

Let us introduce a subspace $\mathcal{H}_{m}$ for a fixed non-negative integer $m$ spanned by the states

$$
\langle 0| C^{2}\left(\theta_{p_{1}}\right) \cdots C^{2}\left(\theta_{p_{m}}\right) C^{3}\left(\theta_{p_{m+1}}\right) \cdots C^{3}\left(\theta_{p_{N}}\right),
$$

where $1 \leq p_{1}<\cdots<p_{m} \leq N, 1 \leq p_{m+1}<\cdots<p_{N} \leq N$ and $p_{j} \neq p_{k}$. It is easy to check that the dimension of the subspace is $\frac{N !}{m !(N-m) !}$ and that the subspace can also be spanned by another set of states

$$
\langle\overline{0}| D_{3}^{2}\left(\theta_{p_{1}}\right) \cdots D_{3}^{2}\left(\theta_{p_{m}}\right), \quad\langle\overline{0}|=\langle 3, \cdots, 3|,
$$

where $1 \leq p_{1}<\cdots<p_{m} \leq N$. Similar to the procedure for deriving (3.17), we have

$$
\left\langle\overline{0}\left|D_{3}^{2}\left(\theta_{p_{1}}\right) \cdots D_{3}^{2}\left(\theta_{p_{m}}\right) D_{2}^{3}\left(\theta_{q_{1}}\right) \cdots D_{2}^{3}\left(\theta_{q_{m^{\prime}}}\right)\right| \overline{0}\right\rangle=\delta_{m, m^{\prime}} \prod_{k=1}^{m} \delta_{p_{k}, q_{k}} f_{m}\left(\theta_{p_{1}}, \cdots, \theta_{p_{m}}\right),
$$

where the state $|\overline{0}\rangle=|3, \cdots, 3\rangle$ and the normalized factor $f_{m}\left(\theta_{p_{1}}, \cdots, \theta_{p_{m}}\right)$ is given by (4.9). The relations (3.13)-(3.14) and the operator decomposition (3.24) enable us to derive that

$$
\begin{aligned}
& \left\langle 0\left|C^{2}\left(\theta_{p_{1}}\right) \cdots C^{2}\left(\theta_{p_{m}}\right) C^{3}\left(\theta_{p_{m+1}}\right) \cdots C^{3}\left(\theta_{p_{N}}\right) D_{2}^{3}\left(u_{1}\right) \cdots D_{2}^{3}\left(u_{m}\right)\right| \overline{0}\right\rangle \\
& =\prod_{\alpha=1}^{m} \prod_{k=m+1}^{N} \sinh \left(u_{\alpha}-\theta_{p_{k}}+\eta\right) g_{m}\left(\theta_{p_{1}}, \cdots, \theta_{p_{m}} \mid u_{1}, \cdots, u_{m}\right)\left\langle 0\left|C^{3}\left(\theta_{1}\right) \cdots C^{3}\left(\theta_{N}\right)\right| \overline{0}\right\rangle \\
& \quad=\prod_{\alpha=1}^{m} \prod_{k=m+1}^{N} \sinh \left(u_{\alpha}-\theta_{p_{k}}+\eta\right) g_{m}\left(\theta_{p_{1}}, \cdots, \theta_{p_{m}} \mid u_{1}, \cdots, u_{m}\right) \prod_{k=1}^{N} a\left(\theta_{k}\right), \quad \text { (D.4) }
\end{aligned}
$$


where the function $g_{m}\left(v_{1}, \cdots, v_{m} \mid u_{1}, \cdots, u_{m}\right)$ is given by (4.8) and we have used the identity: $\left\langle 0\left|C^{3}\left(\theta_{1}\right) \cdots C^{3}\left(\theta_{N}\right)\right| \overline{0}\right\rangle=\prod_{k=1}^{N} a\left(\theta_{k}\right)$.

The corresponding matrix $g$ given by (2.12) allows us to introduce an operator $U(g)$ acting on the Hilbert space as

$$
U(g)=g_{1} g_{2} \cdots g_{N}, \quad\{U(g)\}^{3}=\mathrm{id} .
$$

The invariant property (2.13) of the $R$-matrix and the definition (2.15) of the monodromy matrix $T_{0}(u)$ give rise to the relation

$$
g_{0} T_{0}(u) g_{0}^{-1}=U^{-1}(g) T_{0}(u) U(g),
$$

which implies that

$$
U^{-1}(g) C^{3}(u) U(g)=D_{3}^{2}(u), \quad U^{-1}(g) t(u) U(g)=t(u) .
$$

Direct calculation shows that

$$
\langle 0| U(g)=\langle\overline{0}| .
$$

The invariance (D.7) of the transfer matrix leads to that the state $U(g)|\Psi\rangle$ is also an eigenstate of the transfer matrix with the same eigenvalue, namely,

$$
t(u) U(g)|\Psi\rangle=\Lambda(u) U(g)|\Psi\rangle .
$$

Hence we can simultaneously diagonalize the transfer matrix and the operator $U(g)$, i.e.,

$$
U(g)|\Psi\rangle=\omega^{Z(|\Psi\rangle)}|\Psi\rangle, \quad Z(|\Psi\rangle)=0,1,2 .
$$

Moreover, with the help of the relations (2.5) and (2.6) we can show that

$$
\prod_{j=1}^{N} t\left(\theta_{j}\right)=\left\{\prod_{j=1}^{N} a\left(\theta_{j}\right)\right\} \times U(g),
$$

which gives rise to the identity

$$
\frac{\prod_{j=1}^{N} \Lambda\left(\theta_{j}\right)}{\prod_{j=1}^{N} a\left(\theta_{j}\right)}=\omega^{Z(|\Psi\rangle)} .
$$

The above identity allows one to compute the eigenvalue of the operator $U(g)$ for an eigenstate $|\Psi\rangle$ from the associated Bethe ansatz solution given by (2.18)-(2.27).

The relations (D.7)-(D.9) allow us to derive that

$$
\begin{aligned}
\left\langle\overline{0}\left|D_{3}^{2}\left(\theta_{p_{1}}\right) \cdots D_{3}^{2}\left(\theta_{p_{m}}\right)\right| \Psi\right\rangle & =\left\langle 0\left|C^{3}\left(\theta_{p_{1}}\right) \cdots C^{3}\left(\theta_{p_{m}}\right) U(g)\right| \Psi\right\rangle \\
& \stackrel{(4.5)}{=} \prod_{l=1}^{m} \Lambda\left(\theta_{p_{l}}\right)\langle 0|U(g)| \Psi\rangle \\
& \stackrel{(\mathrm{D} .10)}{=} \omega^{Z(|\Psi\rangle)} \prod_{l=1}^{m} \Lambda\left(\theta_{p_{l}}\right)\langle 0 \mid \Psi\rangle \\
& \stackrel{(\mathrm{D} .12)}{=} \frac{\prod_{j=1}^{N} \Lambda\left(\theta_{j}\right)}{\prod_{j=1}^{N} a\left(\theta_{j}\right)} \times \prod_{l=1}^{m} \Lambda\left(\theta_{p_{l}}\right)\langle 0 \mid \Psi\rangle .
\end{aligned}
$$


Now we are in position to prove (4.7). The relation (4.5) implies that

$$
\begin{aligned}
F_{m}\left(\theta_{p_{1}}, \cdots, \theta_{p_{m}}\right)= & \left\langle 0\left|C^{2}\left(\theta_{p_{1}}\right) \cdots C^{2}\left(\theta_{p_{m}}\right)\right| \Psi\right\rangle \\
= & \frac{\left\langle 0\left|C^{2}\left(\theta_{p_{1}}\right) \cdots C^{2}\left(\theta_{p_{m}}\right) C^{3}\left(\theta_{p_{m+1}}\right) \cdots C^{3}\left(\theta_{p_{N}}\right)\right| \Psi\right\rangle}{\prod_{k=m+1}^{N} \Lambda\left(\theta_{p_{k}}\right)} \\
= & \sum_{\substack{1 \leq p_{1}^{\prime}<\cdots<p_{m}^{\prime} \leq N\\
}} \frac{\left\langle\overline{0}\left|D_{3}^{2}\left(\theta_{p_{1}^{\prime}}\right) \cdots D_{3}^{2}\left(\theta_{p_{m}^{\prime}}\right)\right| \Psi\right\rangle}{f_{m}\left(\theta_{p_{1}^{\prime}}, \cdots, \theta_{p_{m}^{\prime}}\right) \prod_{k=m+1}^{N} \Lambda\left(\theta_{p_{k}}\right)} \\
& \times\left\langle 0\left|C^{2}\left(\theta_{p_{1}}\right) \cdots C^{2}\left(\theta_{p_{m}}\right) C^{3}\left(\theta_{p_{m+1}}\right) \cdots C^{3}\left(\theta_{p_{N}}\right) D_{2}^{3}\left(\theta_{p_{1}^{\prime}}\right) \cdots D_{2}^{3}\left(\theta_{p_{m}^{\prime}}\right)\right| \overline{0}\right\rangle .
\end{aligned}
$$

Substituting the equations (D.4) and (D.13) into the above equation, we finally have the relation (4.7).

Open Access. This article is distributed under the terms of the Creative Commons Attribution License (CC-BY 4.0), which permits any use, distribution and reproduction in any medium, provided the original author(s) and source are credited.

\section{References}

[1] J.M. Maldacena, The large- $N$ limit of superconformal field theories and supergravity, Int. J. Theor. Phys. 38 (1999) 1113 [Adv. Theor. Math. Phys. 2 (1998) 231] [hep-th/9711200] [INSPIRE].

[2] N. Beisert et al., Review of AdS/CFT integrability: an overview, Lett. Math. Phys. 99 (2012) 3 [arXiv:1012.3982] [INSPIRE].

[3] J. Dukelsky, S. Pittel and G. Sierra, Colloquium: Exactly solvable Richardson-Gaudin models for many-body quantum systems, Rev. Mod. Phys. 76 (2004) 643 [nucl-th/0405011] [INSPIRE].

[4] X.-W. Guan, M.T. Batchelor and C. Lee, Fermi gases in one dimension: from Bethe ansatz to experiments, Rev. Mod. Phys. 85 (2013) 1633 [arXiv:1301.6446].

[5] E.K. Sklyanin, Boundary conditions for integrable quantum systems, J. Phys. A 21 (1988) 2375 [inSPIRE].

[6] H. Fan, B.-y. Hou, K.-j. Shi and Z.-x. Yang, Algebraic Bethe ansatz for eight vertex model with general open boundary conditions, Nucl. Phys. B 478 (1996) 723 [hep-th/9604016] [INSPIRE].

[7] R.I. Nepomechie, Bethe ansatz solution of the open XX spin chain with non-diagonal boundary terms, J. Phys. A 34 (2001) 9993 [hep-th/0110081] [INSPIRE].

[8] R.I. Nepomechie, Solving the open XXZ spin chain with nondiagonal boundary terms at roots of unity, Nucl. Phys. B 622 (2002) 615 [hep-th/0110116] [INSPIRE].

[9] R.I. Nepomechie, Functional relations and Bethe ansatz for the XXZ chain, J. Stat. Phys. 111 (2003) 1363 [hep-th/0211001] [INSPIRE].

[10] R.I. Nepomechie, Bethe ansatz solution of the open XXZ chain with nondiagonal boundary terms, J. Phys. A 37 (2004) 433 [hep-th/0304092] [inSPIRE]. 
[11] J. Cao, H.-Q. Lin, K.-J. Shi and Y. Wang, Exact solution of XXZ spin chain with unparallel boundary fields, Nucl. Phys. B 663 (2003) 487.

[12] J. de Gier and P. Pyatov, Bethe ansatz for the Temperley-Lieb loop model with open boundaries, J. Stat. Mech. (2004) P03002 [hep-th/0312235] [INSPIRE].

[13] A. Nichols, V. Rittenberg and J. de Gier, One-boundary Temperley-Lieb algebras in the XXZ and loop models, J. Stat. Mech. (2005) P03003 [cond-mat/0411512] [INSPIRE].

[14] J. de Gier, A. Nichols, P. Pyatov and V. Rittenberg, Magic in the spectra of the XXZ quantum chain with boundaries at $\Delta=0$ and $\Delta=-1 / 2$, Nucl. Phys. B 729 (2005) 387 [hep-th/0505062] [INSPIRE].

[15] W.-L. Yang, Y.-Z. Zhang and M.D. Gould, Exact solution of the XXZ Gaudin model with generic open boundaries, Nucl. Phys. B 698 (2004) 503 [hep-th/0411048] [INSPIRE].

[16] J. de Gier and F.H.L. Essler, Bethe ansatz solution of the asymmetric exclusion process with open boundaries, Phys. Rev. Lett. 95 (2005) 240601 [INSPIRE].

[17] J. de Gier and F.H.L. Essler, Exact spectral gaps of the asymmetric exclusion process with open boundaries, J. Stat. Mech. (2006) P12011 [cond-mat/0609645].

[18] A. Doikou and P.P. Martin, On quantum group symmetry and Bethe ansatz for the asymmetric twin spin chain with integrable boundary, J. Stat. Mech. (2006) P06004 [hep-th/0503019] [INSPIRE].

[19] A. Doikou, The open $X X Z$ and associated models at $q$ root of unity, J. Stat. Mech. (2006) P09010 [hep-th/0603112] [INSPIRE].

[20] Z. Bajnok, Equivalences between spin models induced by defects, J. Stat. Mech. (2006) P06010 [hep-th/0601107] [INSPIRE].

[21] W.-L. Yang, R.I. Nepomechie and Y.-Z. Zhang, Q-operator and T-Q relation from the fusion hierarchy, Phys. Lett. B 633 (2006) 664 [hep-th/0511134] [INSPIRE].

[22] M. Jimbo and T. Miwa, Algebraic analysis of solvable lattice models, CBMS Regional Conference Series in Mathematics, Vol. 85, AMS (1994).

[23] M. Jimbo, K. Miki, T. Miwa and A. Nakayashiki, Difference equations for the correlation functions of the eight-vertex model, J. Phys. A 26 (1993) 2199 [hep-th/9211066] [INSPIRE].

[24] B. Davies, O. Foda, M. Jimbo, T. Miwa and A. Nakayashiki, Diagonalization of the XXZ Hamiltonian by vertex operators, Commun. Math. Phys. 151 (1993) 89 [hep-th/9204064] [INSPIRE].

[25] M. Jimbo, R. Kedem, T. Kojima, H. Konno and T. Miwa, XXZ chain with a boundary, Nucl. Phys. B 441 (1995) 437 [hep-th/9411112] [InSPIRE].

[26] M. Jimbo, R. Kedem, H. Konno, T. Miwa and R. Weston, Difference equations in spin chains with a boundary, Nucl. Phys. B 448 (1995) 429 [hep-th/9502060] [INSPIRE].

[27] P. Baseilhac and T. Kojima, Correlation functions of the half-infinite XXZ spin chain with a triangular boundary, Nucl. Phys. B $\mathbf{8 8 0}$ (2014) 378 [arXiv: 1309.7785] [INSPIRE].

[28] P. Baseilhac and T. Kojima, Form factors of the half-infinite XXZ spin chain with a triangular boundary, J. Stat. Mech. (2014) P09004 [arXiv: 1404.0491] [INSPIRE].

[29] J. Cao, W. Yang, K. Shi and Y. Wang, Off-diagonal Bethe ansatz and exact solution of a topological spin ring, Phys. Rev. Lett. 111 (2013) 137201 [arXiv:1305.7328] [INSPIRE]. 
[30] Y. Wang, W.-L. Yang, J. Cao and K. Shi, Off-diagonal Bethe ansatz for exactly solvable models, Springer Press (2015).

[31] J. Cao, W.-L. Yang, K. Shi and Y. Wang, Off-diagonal Bethe ansatz solution of the XXX spin-chain with arbitrary boundary conditions, Nucl. Phys. B 875 (2013) 152 [arXiv:1306.1742] [INSPIRE].

[32] J. Cao, W.-L. Yang, K. Shi and Y. Wang, Off-diagonal Bethe ansatz solutions of the anisotropic spin-1/2 chains with arbitrary boundary fields, Nucl. Phys. B 877 (2013) 152 [arXiv: 1307.2023] [INSPIRE].

[33] J. Cao, W.-L. Yang, S. Cui, K.-J. Shi and Y. Wang, Spin- $\frac{1}{2}$ XYZ model revisit: general solutions via off-diagonal Bethe ansatz, Nucl. Phys. B 886 (2014) 185 [arXiv:1307.0280] [INSPIRE].

[34] Y.-Y. Li, J. Cao, W.-L. Yang, K. Shi and Y. Wang, Thermodynamic limit and surface energy of the XXZ spin chain with arbitrary boundary fields, Nucl. Phys. B 884 (2014) 17 [arXiv: 1401.3045] [INSPIRE].

[35] J. Cao, W.-L. Yang, K. Shi and Y. Wang, Nested off-diagonal Bethe ansatz and exact solutions of the $\mathrm{su}(n)$ spin chain with generic integrable boundaries, JHEP 04 (2014) 143 [arXiv: 1312.4770] [INSPIRE].

[36] K. Hao et al., Exact solution of the Izergin-Korepin model with general non-diagonal boundary terms, JHEP 06 (2014) 128 [arXiv:1403.7915] [INSPIRE].

[37] X. Zhang et al., Retrieve the Bethe states of quantum integrable models solved via off-diagonal Bethe ansatz, J. Stat. Mech. (2015) P05014 [arXiv:1407.5294] [INSPIRE].

[38] X. Zhang et al., Bethe states of the XXZ spin- $\frac{1}{2}$ chain with arbitrary boundary fields, Nucl. Phys. B 893 (2015) 70 [arXiv:1412.6905] [INSPIRE].

[39] P. Baseilhac, The q-deformed analogue of the Onsager algebra: beyond the Bethe ansatz approach, Nucl. Phys. B 754 (2006) 309 [math-ph/0604036] [INSPIRE].

[40] P. Baseilhac and K. Koizumi, Exact spectrum of the XXZ open spin chain from the q-Onsager algebra representation theory, J. Stat. Mech. (2007) P09006 [hep-th/0703106] [INSPIRE].

[41] P. Baseilhac and S. Belliard, Generalized q-Onsager algebras and boundary affine Toda field theories, Lett. Math. Phys. 93 (2010) 213 [arXiv:0906.1215] [InSPIRE].

[42] P. Baseilhac and S. Belliard, The half-infinite XXZ chain in Onsager's approach, Nucl. Phys. B $\mathbf{8 7 3}$ (2013) 550 [arXiv:1211.6304] [INSPIRE].

[43] H. Frahm, A. Seel and T. Wirth, Separation of variables in the open XXX chain, Nucl. Phys. B 802 (2008) 351 [arXiv:0803.1776] [INSPIRE].

[44] H. Frahm, J.H. Grelik, A. Seel and T. Wirth, Functional Bethe ansatz methods for the open XXX chain, J. Phys. A 44 (2011) 015001 [arXiv:1009.1081] [InSPIRE].

[45] S. Niekamp, T. Wirth and H. Frahm, The XXZ model with anti-periodic twisted boundary conditions, J. Phys. A 42 (2009) 195008 [arXiv:0902.1079] [InSPIRE].

[46] G. Niccoli, Non-diagonal open spin-1/2 XXZ quantum chains by separation of variables: complete spectrum and matrix elements of some quasi-local operators, J. Stat. Mech. (2012) P10025 [arXiv:1206.0646] [INSPIRE].

[47] G. Niccoli, Antiperiodic spin-1/2 XXZ quantum chains by separation of variables: complete spectrum and form factors, Nucl. Phys. B 870 (2013) 397 [arXiv: 1205.4537] [INSPIRE]. 
[48] G. Niccoli, An antiperiodic dynamical six-vertex model: I. Complete spectrum by SOV, matrix elements of the identity on separate states and connections to the periodic eight-vertex model, J. Phys. A 46 (2013) 075003 [arXiv: 1207.1928] [INSPIRE].

[49] N. Kitanine, J.-M. Maillet and G. Niccoli, Open spin chains with generic integrable boundaries: Baxter equation and Bethe ansatz completeness from separation of variables, J. Stat. Mech. (2014) P05015 [arXiv:1401.4901] [INSPIRE].

[50] S. Belliard and N. Crampé, Heisenberg XXX model with general boundaries: eigenvectors from algebraic Bethe ansatz, SIGMA 9 (2013) 072 [arXiv: 1309.6165] [INSPIRE].

[51] S. Belliard, Modified algebraic Bethe ansatz for XXZ chain on the segment - I: triangular cases, Nucl. Phys. B 892 (2015) 1 [arXiv:1408.4840] [INSPIRE].

[52] S. Belliard and R.A. Pimenta, Modified algebraic Bethe ansatz for XXZ chain on the segment - II: general cases, Nucl. Phys. B 894 (2015) 527 [arXiv: 1412.7511] [InSPIRE].

[53] J. Avan, S. Belliard, N. Grosjean and R.A. Pimenta, Modified algebraic Bethe ansatz for XXZ chain on the segment - III: proof, Nucl. Phys. B 899 (2015) 229 [INSPIRE].

[54] X. Zhang, J. Cao, W.-L. Yang, K. Shi and Y. Wang, Exact solution of the one-dimensional super-symmetric $t-J$ model with unparallel boundary fields, J. Stat. Mech. (2014) P04031 [arXiv: 1312.0376] [INSPIRE].

[55] X. Zhang et al., Bethe ansatz for an AdS/CFT open spin chain with non-diagonal boundaries, JHEP 10 (2015) 133 [arXiv:1507.08866] [INSPIRE].

[56] E.K. Sklyanin, The quantum Toda chain, Lect. Notes Phys. 226 (1985) 196 [INSPIRE].

[57] E.K. Sklyanin, Goryachev-Chaplygin top and the inverse scattering method, J. Sov. Math. 31 (1985) 3417 [INSPIRE].

[58] E.K. Sklyanin, Separation of variables - new trends, Prog. Theor. Phys. Suppl. 118 (1995) 35 [solv-int/9504001] [INSPIRE].

[59] K. Hao et al., Exact solution of an $\mathrm{su}(n)$ spin torus, arXiv:1601.04389 [INSPIRE].

[60] V. Chari and A. Pressley, A guide to quantum groups, Cambridge University Press, Cambridge U.K. (1994).

[61] J.H.H. Perk and C.L. Schultz, New families of commuting transfer matrices in q state vertex models, Phys. Lett. A 84 (1981) 407 [INSPIRE].

[62] J.H.H. Perk and C.L. Schultz, Families of commuting transfer matrices in q-state vertex models, in Non-linear integrable systems - classical theory and quantum theory, M. Jimbo and T. Miwa eds., World Scientific (1983), pp. 135-152.

[63] C.L. Schultz, Eigenvectors of the multi-component generalization of the six-vertex model, Physica A 122 (1983) 71.

[64] J.H.H. Perk and H. Au-Yang, Yang-Baxter equations, in Encyclopedia of mathematical physics, J.-P. Françoise, G.L. Naber and T.S. Tsun eds., Academic Press (2006) [math-ph/0606053].

[65] V.V. Bazhanov, Trigonometric solution of triangle equations and classical Lie algebras, Phys. Lett. B 159 (1985) 321 [INSPIRE].

[66] M. Jimbo, Quantum R matrix for the generalized Toda system, Commun. Math. Phys. 102 (1986) 537 [InSPIRE]. 
[67] R.I. Nepomechie, Boundary quantum group generators of type A, Lett. Math. Phys. 62 (2002) 83 [hep-th/0204181] [INSPIRE].

[68] M.T. Batchelor, R.J. Baxter, M.J. O'Rourke and C.M. Yung, Exact solution and interfacial tension of the six vertex model with antiperiodic boundary conditions, J. Phys. A 28 (1995) 2759 [hep-th/9502040] [INSPIRE].

[69] V.G. Drinfel'd, On constant quasiclassical solutions of the Yang-Baxter quantum equation, Sov. Math. Dokl. 28 (1983) 667.

[70] J.M. Maillet and J. Sanchez de Santos, Drinfel'd twists and algebraic Bethe ansatz, q-alg/9612012 [INSPIRE].

[71] T.D. Albert, H. Boos, R. Flume and K. Ruhlig, Resolution of the nested hierarchy for rational $\mathrm{sl}(n)$ models, J. Phys. A 33 (2000) 4963 [nlin/0002027] [INSPIRE].

[72] T.D. Albert and K. Ruhlig, Polarization free generators for the Belavin model, J. Phys. A 34 (2001) 1569 [nlin/0007020] [InSPIRE].

[73] W.-L. Yang, Y.-Z. Zhang and S.-Y. Zhao, Drinfeld twists and algebraic Bethe ansatz of the supersymmetric model associated with $U_{q}(\operatorname{gl}(M \mid N))$, Commun. Math. Phys. 264 (2006) 87 [hep-th/0503003] [INSPIRE].

[74] E.K. Sklyanin, Separation of variables in the quantum integrable models related to the Yangian y[sl(3)], J. Math. Sci. 80 (1996) 1861 [hep-th/9212076] [INSPIRE].

[75] H.J. de Vega and E. Lopes, Exact solution of the Perk-Schultz model, Phys. Rev. Lett. 67 (1991) 489 [INSPIRE].

[76] E. Lopes, Exact solution of the multi-component generalized six-vertex model, Nucl. Phys. B 370 (1992) 636 [inSPIRE].

[77] V.E. Korepin, N.M. Bogoliubov and A.G. Izergin, Quantum inverse scattering method and correlation function, Cambridge University Press, Cambridge U.K. (1993).

[78] S.-Y. Zhao, W.-L. Yang and Y.-Z. Zhang, Determinant representation of correlation functions for the supersymmetric $t-J$ model, Commun. Math. Phys. 268 (2006) 505 [hep-th/0511028] [INSPIRE].

[79] S.-Y. Zhao, W.-L. Yang and Y.-Z. Zhang, On the construction of correlation functions for the integrable supersymmetric fermion models, Int. J. Mod. Phys. B 20 (2006) 505 [hep-th/0601065] [INSPIRE].

[80] S. Belliard, S. Pakuliak, E. Ragoucy and N.A. Slavnov, Bethe vectors of quantum integrable models with GL(3) trigonometric R-matrix, SIGMA 9 (2013) 058 [arXiv:1304.7602] [INSPIRE].

[81] S. Belliard, S. Pakuliak, E. Ragoucy and N.A. Slavnov, Form factors in SU(3)-invariant integrable models, J. Stat. Mech. (2013) P04033 [arXiv:1211.3968] [INSPIRE].

[82] M.J. Matins and P.B. Ramos, The quantum inverse scattering method for Hubbard-like models, Nucl. Phys. B 522 (1998) 413. 